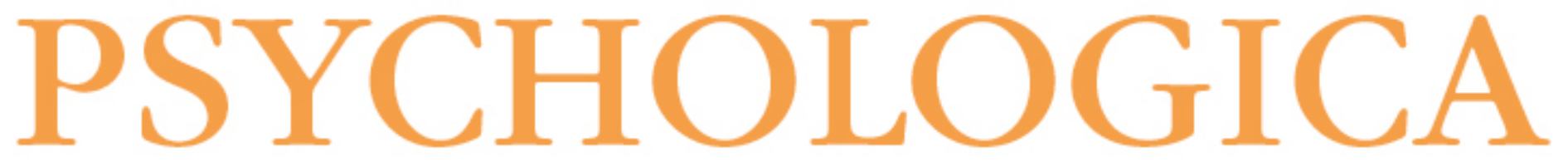

\title{
Suporte social parental e prevenção na primeira infância em contextos de adversidade: a perspetiva de profissionais de educação de infância e saúde mental no Brasil
}

Autor(es): $\quad$ Novaes, Marcelly de Brito; Gaspar, Maria Filomena

Publicado por: Imprensa da Universidade de Coimbra

URL persistente:

URl:http://hdl.handle.net/10316.2/40767

DOI:

DOI:https://doi.org/10.14195/1647-8606_59-1_7

Accessed : $\quad$ 26-Apr-2023 13:47:57

A navegação consulta e descarregamento dos títulos inseridos nas Bibliotecas Digitais UC Digitalis, UC Pombalina e UC Impactum, pressupõem a aceitação plena e sem reservas dos Termos e Condições de Uso destas Bibliotecas Digitais, disponíveis em https://digitalis.uc.pt/pt-pt/termos.

Conforme exposto nos referidos Termos e Condições de Uso, o descarregamento de títulos de acesso restrito requer uma licença válida de autorização devendo o utilizador aceder ao(s) documento(s) a partir de um endereço de IP da instituição detentora da supramencionada licença.

Ao utilizador é apenas permitido o descarregamento para uso pessoal, pelo que o emprego do(s) título(s) descarregado(s) para outro fim, designadamente comercial, carece de autorização do respetivo autor ou editor da obra.

Na medida em que todas as obras da UC Digitalis se encontram protegidas pelo Código do Direito de Autor e Direitos Conexos e demais legislação aplicável, toda a cópia, parcial ou total, deste documento, nos casos em que é legalmente admitida, deverá conter ou fazer-se acompanhar por este aviso. 
VOLUME 5

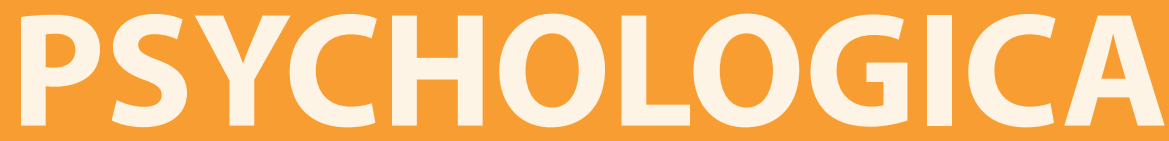

IMPRENSA DA UNIVERSIDADE DE COIMBRA

COIMBRA UNIVERSITY PRESS

FACULDADE DE PSICOLOGIA E DE CIÊNCIAS

DA EDUCAÇÃO DA UNIVERSIDADE DE COIMBRA 


\title{
Suporte social parental e prevenção na primeira infância em contextos de adversidade: A perspetiva de profissionais de educação de infância e saúde mental no Brasil
}

\author{
Marcelly de Brito Novaes ${ }^{1}$ e Maria Filomena Gaspar ${ }^{2}$
}

Parental social support and early childhood prevention in contexts of adversity: The perspective of early years' educators and mental health professionals in Brazil

\begin{abstract}
This qualitative and exploratory study aims to understand the perceptions of professionals, who work in a day care centre and a mental health service for children (Psychosocial Attention Centre for Childhood - CAPSi), about the formal parental social support they offer within contexts of adversity. It also aims to understand the perceptions of parental exercise in Brazilian culture by those professionals.

The participant institutions serve families in adversity settings or residents of favelas in Rio de Janeiro city, Brazil. Semi structured interviews and participant observation were done during nine weeks. Seventeen child care professionals (helpers) were interviewed, six in the day care centre and 11 in the CAPSi. The helpers relate the difficulties on the intervention process to the families' socioeconomic context. Negative and coercive parental attitudes are considered to be normal by the professionals within the scope of the Brazilian culture. Professional social support is a need for helpers of formal parental support to families in socioeconomic' conditions of adversity in Brazil.
\end{abstract}

Keywords: adversity; early childhood; positive parenting; prevention; parental social support

1 Doutorada em Psicologia pelo curso de doutoramento interuniversitário em Psicologia da Família e Intervenção Familiar, da Universidade de Coimbra e da Universidade de Lisboa. Email: marcelly.de.brito.novaes@gmail.com

2 Professora Associada da Faculdade de Psicologia e de Ciências da Educação da Universidade de Coimbra. Email: ninigaspar@fpce.uc.pt 


\section{Resumo}

Este estudo, qualitativo e exploratório, tem como objetivo conhecer as perceções de profissionais que trabalham em creche e em serviços de saúde mental para a infância (Centro de Atenção Psicossocial para a Infância - CAPSi) sobre as suas funções de suporte social parental em contextos de adversidade. Ambiciona, simultaneamente, identificar as representações sobre parentalidade subjacentes a essas perceções.

As instituições participantes servem famílias moradoras em favelas ou em condições de adversidade na cidade do Rio de Janeiro, no Brasil. Utilizaram-se entrevistas semiestruturadas e observação participante durante nove semanas. Foram entrevistados 17 profissionais cuidadores de crianças, sendo seis na creche e 11 no CAPSi. Dificuldades na intervenção são atribuídas, pelos profissionais, ao contexto socioeconómico das famílias. Atitudes parentais negativas e coercitivas são normalizadas pelos profissionais no âmbito da cultura brasileira. Conclui-se pela necessidade de suporte social ao profissional de suporte social parental formal à família em condição de adversidade socioeconómica no Brasil.

Palavras-chave: adversidade; primeira infância; parentalidade positiva; prevenção; suporte social parental

\section{INTRODUÇÃO}

A teoria da mudança (Center on the Developing Child at Harvard University [CDCHU], 2010) propõe o suporte social familiar do Estado e o endereçamento das fontes de "stresse tóxico" das famílias como forma de potenciar o desenvolvimento pleno de crianças que vivem em situação de adversidade. Especificamente na primeira infância, políticas e ações de suporte aos profissionais que trabalham com as crianças na comunidade são consideradas um investimento em formação de recursos humanos a médio e longo prazo (Cunha \& Heckman, 2007; Doyle, Harmon, Heckman, \& Tremblay, 2009), além de contribuírem para a preparação da criança para a escola (Araújo et al., 2011). O suporte social é definido como as relações sociais que disponibilizam recursos materiais e interpessoais que são de valor para o recetor do suporte (Thompson, 1995). O suporte formal é dado pelo Estado e por instituições ou profissionais especialistas no suporte social para a família (Thompson \& Goodvin, 2015). Esses profissionais estão na comunidade, envolvidos no processo educativo e desenvolvimental da criança, seja na educação, saúde ou assistência social. Entre as intervenções destes profissionais, no suporte social familiar, encontra-se a aplicação de intervenções sistemáticas e baseadas em 
evidências para o desenvolvimento de uma atitude parental positiva. Considera-se a parentalidade positiva como uma atitude parental que procura garantir a satisfação dos melhores interesses para a criança, de forma não violenta, e promove a capacitação da criança ao permitir o pleno desenvolvimento das suas potencialidades (Abreu-Lima et al., 2010; Child on Europe Report [CER], 2011, 2013; Cruz, 2005).

No contexto da conceção de suporte social de Thompson e Goodvin (2015), no âmbito da parentalidade, são identificadas cinco funções de suporte social parental. A primeira função é de suporte emocional e engloba a perceção do indivíduo de empatia, compreensão e afirmação emocional das dificuldades vivenciadas. A segunda função é de suporte instrumental e está relacionada aos diversos tipos de suporte no âmbito do dia a dia familiar seja transporte, financeiro, de acesso a informação e serviços ou de cuidado direto com a criança. A terceira função é de orientação e aconselhamento parental e está relacionada ao processo educativo da criança. A quarta função é de aquisição de habilidades parentais, logo é o aspeto do suporte que lida com a capacitação das figuras parentais com práticas parentais de promoção do desenvolvimento ajustado da criança. A quinta função é a de monitorização social. Nesta função destaca-se a influência protetora da rede de suporte social para o bem-estar da figura parental e da criança, seja para deteção de necessidades específicas de suporte, seja para promover ações positivas e partilhadas.

A natureza da relação formal de suporte social tem sido investigada a partir de perspetivas distintas dessa relação, nomeadamente, as perceções do profissional das expectativas da oferta e aceitação do suporte social pela figura parental (NielsenBerg, Solhein, Belsky, \& Wichstrom, 2011; Thompson, 2011), além das sensações e emoções positivas e negativas relacionadas ao suporte social (Raikes \& Thompson, 2005; Thompson, 2015), e componentes do estabelecimento e manutenção da relação entre o profissional que oferece suporte e o recetor desse mesmo suporte (Thompson \& Goodvin, 2015). O modelo de sustentabilidade para intervenções de suporte social parental que propomos, representado na Figura 1, apresenta uma possibilidade de avaliação contextual do suporte social formal familiar. Neste modelo, devido à deterioração da rede potencial de suporte social parental e afastamento do próprio recetor da potencial rede de suporte, somente o suporte formal possibilita que a condição social familiar não se agrave. O treinamento em habilidades parentais contribui para a contenção do "stresse tóxico" e para o desenvolvimento de fatores de prevenção contextual, por exemplo, ao ampliar a rede de suporte social formal percebida pelo indivíduo. Também atua como fator de prevenção nas relações do núcleo familiar, ao alterar as práticas de socialização da criança e convergir as ações da intervenção para a normatização das relações intrafamiliares (CDCHU, 2010; Novaes, 2015) (cf. Figura 1). Compreende-se que redes de suporte social parental formal que são familiares às figuras parentais, ou seja, de conhecimento 
prévio à necessidade de suporte, ampliam as possibilidades de acesso a intervenções sobre o exercício parental e ações de contenção de "stresse tóxico" e prevenção do agravamento do risco social, conforme observado em alguns estudos (Raikes \& Thompson, 2005; Thompson \& Goodvin, 2015) e apresentado na Figura 1.

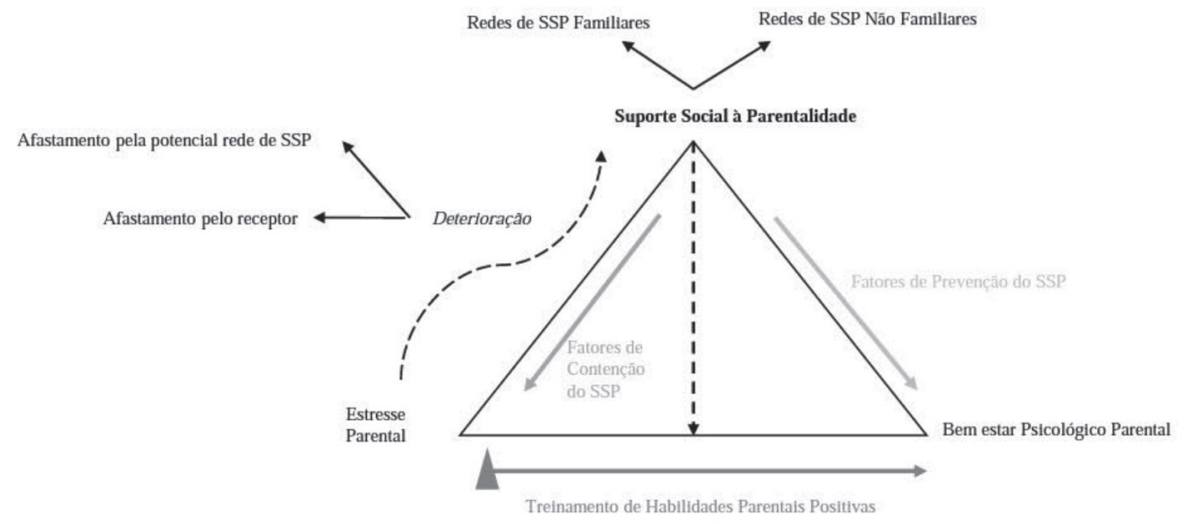

Figura 1. Modelo de sustentabilidade de intervenções de suporte social parental.

Várias investigações debruçaram-se sobre o estabelecimento e desenvolvimento da relação no suporte social formal entre o profissional e a figura parental (Axberg \& Broberg, 2012; McGilloway et al., 2012; Morawska et al., 2011; Respler-Herman, Mowder, Yasik, \& Shamah, 2012). Investigações para identificação de influências negativas e positivas no estabelecimento dessa relação (Nielsen-Berg et al., 2011; Thompson \& Goodvin, 2015) identificaram perspetivas dissonantes sobre o exercício parental e expetativas relacionadas com a parentalidade entre profissionais e figuras parentais.

No que se refere ao exercício parental, a abordagem cultural (Bornstein, 2012; Harkeness \& Super, 2002) permite a compreensão do desenvolvimento da parentalidade na cultura em resultado da interação de cinco conceitos: as etnoteorias, as crenças parentais, as cognições parentais, as práticas parentais e as práticas de socialização da criança. As etnoteorias referem-se às perceções sobre a parentalidade partilhadas na cultura e influenciadas pela etnia e contexto socioeconómico do indivíduo. As crenças parentais são desenvolvidas a partir de etnoteorias e constituem valores relacionados ao exercício parental e ao desenvolvimento da criança. As cognições parentais são as ações de pensamento direcionadas pelas figuras parentais para concretização das crenças parentais. As práticas parentais são as práticas de cuidado e socialização da criança, sendo que as práticas de socialização estão relacionadas com a circulação social e ajustamento da criança na comunidade. 
Especificamente, esse estudo pretende compreender a perspetiva de profissionais sobre o suporte social oferecido às famílias, que vivem em condições de adversidade e com filhos na primeira infância, considerando-se crianças com desenvolvimento típico e atípico, especificamente crianças diagnosticadas com Transtorno do Espetro Autista (TEA) (American Psychiatric Association [APA], 2013), em equipamentos públicos de acesso diário ou semanal. Nesse estudo, o grupo de profissionais de suporte parental no desenvolvimento atípico restringiu-se aos que atuam no tratamento e inserção psicossocial. Justifica-se essa escolha devido a elaboração, no Brasil, de diretrizes específicas para educação e inclusão psicossocial de famílias com crianças diagnosticadas no TEA (Brasil, 2009, 2013; Lima, Couto, Delgado, \& Oliveira, 2014).

\section{MÉTODo}

Este estudo, qualitativo e exploratório, teve como objetivo identificar as perceções de profissionais que trabalham com crianças e suas famílias em condições de adversidade, no contexto brasileiro, sobre o suporte social parental e sobre a parentalidade na cultura brasileira. Nomeadamente, procura-se compreender as componentes culturais da parentalidade na perspetiva dos profissionais e identificar as funções de suporte social parental no suporte que é oferecido e recebido pelas famílias com crianças na primeira infância com e sem perturbações de desenvolvimento. Com esse objetivo recorreu-se a entrevistas semiestruturadas e observação participante em duas instituições de suporte social familiar.

\section{Participantes}

Duas instituições participaram da investigação, conforme conveniência e disponibilidade para participação no estudo após carta convite. Nomeadamente, uma creche conveniada à Prefeitura do Rio de Janeiro e um CAPSi, serviço público do Ministério da Saúde do Brasil para encaminhamento e tratamento de crianças em saúde mental. Ambas as instituições estão localizadas em zona urbana, na zona sul da cidade do Rio de Janeiro, no Brasil. A zona sul do Rio de Janeiro é identificada como zona de maior poder socioeconómico na capital do estado do Rio de Janeiro (Borges, Magalhães, \& Féres-Carneiro, 2015; Novaes, 2015; Ramos, Seidl-de-Moura, \& Pessoa, 2013).

Participaram da investigação 17 profissionais, sendo seis da creche e 11 do CAPSi. Todos os profissionais são do sexo feminino. Na creche, a média de idade era 30 anos, três educadoras infantis possuíam graduação em Pedagogia e especializações 
lato sensu na área de educação e uma educadora estava cursando a graduação em Pedagogia. As duas outras educadoras infantis possuíam ensino médio completo. No Capsi, a média de idade dos profissionais era de 43 anos. Somente um profissional em saúde mental possuía ensino técnico, nomeadamente em Enfermagem. Os demais possuíam graduação e especialização stricto sensu ou lato sensu. Dois eram graduados em Medicina, tendo uma com residência médica em psiquiatria infantil e outra em formação na mesma área. Quatro eram graduadas em Pedagogia, uma em Assistência Social e uma em Psicologia.

\section{Instrumento e dimensões avaliadas}

Utilizou-se a técnica da entrevista semiestruturada e observação participante. O guião de entrevista foi desenvolvido a partir do modelo de suporte social (Thompson \& Goodvin, 2015), da conceção cultural da parentalidade (Bornstein, 2012; Harkness \& Super, 2002) e da teoria da mudança (CDCHU, 2010). As perguntas foram organizadas em blocos. O bloco I engloba os dados sociodemográficos do profissional e da instituição. O bloco II diz respeito aos fatores culturais do exercício parental. O bloco III refere-se a perceção de redes informais de suporte social parental. O bloco IV avalia as perceções sobre o suporte social parental formal e informal e a compreensão das expectativas das relações de suporte à parentalidade no âmbito das cinco funções de suporte social aplicadas à parentalidade. $\mathrm{O}$ bloco $\mathrm{V}$ é referente ao exercício parental em condições de adversidade social. O guião foi revisto por uma especialista em Psicologia da Família e na implementação de programas de formação parental. Foram conduzidas três entrevistas piloto para aferir a clareza e adequação das perguntas.

\section{Materiais}

Recorreu-se a utilização do telefone celular com o guião no ecrã. Na nossa opinião a informalidade e familiaridade do aparelho celular nos contextos diários informais, em comparação ao guião impresso, contribui para o envolvimento ativo dos profissionais nas entrevistas. Além do celular recorremos a um gravador para as gravações de áudio das entrevistas.

\section{Procedimento de recolha de amostra}

A pesquisadora e primeira autora desse artigo realizou observação participante, em período integral, por nove semanas em ambas as instituições. A duração de cada 
entrevista variou entre 50 e 120 minutos e todas as entrevistas foram realizadas nas instituições participantes em horário laboral.

\section{Cumprimento dos princípios éticos}

O projeto da investigação foi submetido e aprovado pelo Comitê de Ética de Universidade na cidade do Rio de Janeiro, conforme indicado pelos procedimentos éticos no Brasil para pesquisas com seres humanos. Para participação na investigação todos os participantes assinaram termo de consentimento livre e esclarecido.

\section{Análise dos dados}

O tratamento da narrativa foi realizado através de análise temática (Pêcheux, 1983) com o auxílio do software de análise qualitativa NVivo. Para análise, foram utilizadas as teorias centrais de construção do enquadramento teórico da investigação e do guião de entrevista, nomeadamente, a teoria da mudança (CDCHU, 2010) e abordagem do suporte social (Thompson \& Goodvin, 2015) no âmbito de identificação de fatores do contexto socioeconómico que atuam sobre a perceção do suporte social parental (SSP). A abordagem cultural da parentalidade (Bornstein, 2012; Harkness \& Super, 2002) foi utilizada para análise das representações de parentalidade dos profissionais na cultura brasileira.

Inicialmente as categorias foram identificadas conforme os blocos do guião de entrevista. Entretanto, ao longo do processo de tratamento da narrativa, observou-se que sobre o suporte social parental e o exercício parental encontraram-se perceções de aspetos comuns e distintos entre profissionais e entre as instituições, o que permitiu o desenvolvimento das categorias no processo da análise temática, conforme consta na literatura (Braum \& Clarke, 2006; Miller \& Crabtree, 2004; Rank, 2004; Zimmerman \& Sprague, 2004). Nomeadamente, na categorização final foram designadas seis categorias centrais, com 35 subcategorias, além de categorias menores. A primeira categoria central foi designada como "Redes formais de suporte social parental", e possui quatro subcategorias: "Legislação sobre a parentalidade", "Modelos de suporte formal à parentalidade", "Rede de suporte social parental formal e Estado brasileiro" e "Rede de suporte social parental na comunidade". A segunda categoria central foi designada como "Redes informais de suporte social parental" e tem seis subcategorias: "Amigos da figura parental", "Colegas de trabalho da figura parental", "Figuras comunitárias ou do bairro", "Família estendida", "Pares parentais com filho ou filha na mesma idade" e "Pares crianças". A terceira categoria central foi designada como 
"Funções do suporte social parental", tendo ficado constituída por seis subcategorias, especificamente: "Aconselhamento e orientação parental", "Suporte emocional”, "Suporte instrumental", "Monitoramento social", "Treinamento de habilidades parentais" e "Componentes funcionais do suporte social parental'. A quarta categoria central foi designada como "Intervenções familiares e comunitárias no Brasil', tendo sido composta por duas subcategorias "Inovação e tecnologias de intervenção familiar e comunitária" e "Intervenções familiares e comunitárias disponíveis". A quinta categoria central foi designada como "Adversidade e parentalidade" e ficou constituída por 10 subcategorias: "Dificuldades percebidas na educação da criança", "Fatores de contenção do stresse na criança", "Fatores de desenvolvimento do stresse", "Incentivo para procurar suporte social parental", "Migração familiar", "Parentalidades de riscos", "Perceção atual da rede de suporte social parental em situações de adversidade", "Pobreza no desenvolvimento familiar", "Sintomas de stresse", "Violência intrafamiliar". A sexta categoria central foi designada como "Componentes culturais da parentalidade" e foi composta por sete subcategorias, a saber: "Ambiente familiar', "Cultura e exercício parental”, "Etnoteorias parentais", "Crenças parentais", "Cognições parentais", "Práticas parentais" e "Práticas de socialização da criança". A análise e categorização ocorreu conforme os objetivos da investigação, de modo que a articulação entre categorias centrais e subcategorias no processo de análise procura a identificação de fatores culturais sobre a parentalidade e de funções do suporte social parental. O contexto socioeconómico e perceções relacionadas ao suporte social parental formal e informal são compreendidos no âmbito de acessibilidade ao suporte social necessário e alteração da perceção do "stresse tóxico" pela família, como é possível ver na análise e a partir do Modelo de Sustentabilidade da Intervenção em Suporte Social Parental proposto (cf. Figura 1).

Conforme descrito em alguns estudos, como o de Hutchinson, Johnson e Breckon (2010) sobre a utilização de softwares de análise qualitativa, nesta investigação, durante o processo de análise dos dados, a utilização do software NVivo permitiu ampla comparação entre as diferentes categorias centrais e comparação de frequência de apresentação no discurso das subcategorias. Desse modo foi possível identificar potenciais possibilidades de intervenção no âmbito da prevenção e necessidades de suporte social parental formal no contexto escolar, nomeadamente no contexto de prevenção em saúde mental familiar e comunitária.

\section{RESULTADOS}

Os profissionais relataram que a maioria das famílias que recorre às suas instituições vive em condições de adversidade em favelas da região, onde, apesar de não 
ter acesso a serviços de saneamento básico, possui acesso a áreas de lazer públicas, como praia e praças, além de serviços de transporte e mercado de trabalho. Essa situação foi exposta em contraponto a dificuldades de acessibilidade desses itens públicos por famílias que vivem em condições de pobreza em favelas da zona norte da cidade. Relataram, tanto na creche como no CAPSi, situações de frequência espaçada de crianças moradoras em favelas na instituição devido a situações de violência rotineira na vizinhança da habitação familiar, tais como tiroteios. Adicionalmente, foram citados, pelos profissionais, sintomas de stresse frequentes observados em crianças que residem no local de trabalho da figura parental, quando essa é trabalhadora doméstica ou de condomínio residencial. Os sintomas identificados são alta vigilância, dificuldades de aprendizagem e dificuldades de regulação emocional. Observam-se, assim, sintomas de stresse na criança relacionados ao contexto de pobreza e condições de trabalho de uma das figuras parentais.

Sobre o desenvolvimento da parentalidade na cultura brasileira, os profissionais relatam etnoteorias positivas da figura parental feminina, compreendidas como colaborativas para a educação da criança e inclusão social familiar, especificamente: a oferta de atenção, o carinho e o interesse pelo desempenho académico da criança. Especificamente sobre a figura parental masculina, diversos profissionais observaram a ausência ou indisponibilidade frequente em contexto escolar ou de cuidar da criança. Logo, é compreendida pelos profissionais como etnoteoria positiva sobre a figura parental masculina, a presença atuante em práticas de cuidado com a criança em seus contextos de circulação social.

Sobre etnoteorias negativas relacionadas à parentalidade, compreendidas como negativas devido à não contribuição para o desenvolvimento da criança, foram citadas práticas parentais negativas e necessidade de intervenção parental para alteração de crenças e cognições parentais negativas, ou seja, expetativas negativas de desenvolvimento da criança e de suas possibilidades de educação e desenvolvimento pleno de potencialidades. Percebe-se que cogniçõos parentais negativas, devido a crenças parentais e objetivos de socialização da criança com perturbações do desenvolvimento (e.g., autismo), podem atuar como stressores na vinculação nos primeiros anos de vida entre criança e figura parental. No que se refere às crianças, os profissionais apresentam etnoteorias diferentes para crianças com desenvolvimento típico e atípico (com perturbação do desenvolvimento; e.g., autismo). No desenvolvimento típico, etnoteorias positivas percebidas nas atitudes das crianças, como espontaneidade, alegria, pró-atividade e disponibilidade para ser amiga foram citadas. Já no atípico, a diminuição da presença de sintomas relacionados ao transtorno foi citada como etnoteoria positiva sobre a criança. Observam-se etnoteorias negativas sobre as crianças, tais como as que associam características de timidez e agressividade a perturbações na socialização. 
Sobre a identificação de funções do suporte social parental exercidas no CAPSi e na creche, observa-se que na creche, a função de aconselhamento e orientação oferecidos às famílias foi descrita como exercida quando são identificadas, pelo profissional, atitudes negativas da criança, como agressões físicas aos pares ou ao próprio profissional, choro frequente ou isolamento do grupo. A intervenção familiar realizada, geralmente, é por meio da comunicação à figura parental sobre as atitudes da criança no contexto escolar, conforme recomenda a política de Educação Infantil do país (Brasil, 2009). No CAPSi, sobre essa mesma função de aconselhamento parental na relação entre profissional e figura parental, são descritos relatos de sensação de insegurança no exercício parental.

Sobre a perceção dos profissionais da creche como parte da rede de suporte emocional formal familiar e expetativas com relação ao suporte emocional oferecido, as educadoras de infância atribuíram a procura das figuras parentais de suporte emocional à relação estabelecida, com diálogos frequentes sobre a criança e práticas educativas no contexto escolar. Já no CAPSi, o procedimento da porta de entrada, caraterizado pela receção da família na instituição com entrevista com o profissional, para identificação e endereçamento adequado das necessidades de intervenção em saúde mental para a família, atua como ferramenta de suporte emocional para a família no momento de crise e chegada ao serviço, constituindo-se como fator contentor de stresse para as famílias.

A escola (jardim de infância) é então descrita por alguns profissionais como vista pelas figuras parentais no papel de suporte no cuidado com a criança para o exercício laboral da família. Percebe-se a necessidade de orientação parental sobre o processo educativo e de desenvolvimento psicossocial da criança no espaço físico e cronológico da educação infantil. Sobre benefícios financeiros, observa-se que a alteração na perceção da rede de suporte social parental formal é realizada por meio de intervenção para o reconhecimento da necessidade de suporte instrumental no âmbito financeiro pela família. Sendo então a busca por benefício financeiro por famílias, em condição de adversidade, resultado da ampliação da rede de suporte social parental, conforme proposto no Modelo de Sustentabilidade de Intervenções de Suporte Social Parental (e.g., Figura 1) e da política de Assistência Social para a infância no Brasil (Colin, Pereira, \& Gonelli, 2013; Novaes \& Gaspar, 2015). Entretanto alguns profissionais referiram esse fenómeno como negativo para a intervenção familiar.

No âmbito da natureza da relação formal de suporte social parental, um aspeto citado pelos participantes foi a saúde psicológica do profissional. A eficácia das intervenções com as figuras parentais é relacionada pelos profissionais com a supervisão e disponibilidade oferecida pelos pares profissionais no exercício do suporte social parental. Sobre as sensações advindas da oferta de suporte, essas são um indicador de sobrecarga no trabalho e possível burnout do profissional ou necessidade de 
treinamento para oferta de suporte social formal, seja para o estabelecimento, seja para a manutenção da relação de suporte, conforme indicam alguns estudos (NielsenBerg et al., 2011; Novaes \& Gaspar, 2015; Thompson \& Goodvin, 2015). Conforme a abordagem do suporte social e a identificação das funções de suporte social para a parentalidade, presentes na perceção dos profissionais, foram identificadas funções de suporte ao profissional que visam a sua capacitação. A Figura 2 procura representar esse suporte ao profissional de suporte social parental no exercício das suas diferentes funções. Esse modelo de suporte social profissional ao profissional que disponibiliza suporte social parental pretende, a partir dos dados obtidos, direcionar a perceção da instituição para a necessidade de suporte institucional e por meio de políticas públicas e ações para formação do profissional de suporte social às famílias em condição de adversidade. Sendo assim, observa-se que na relação de suporte social o profissional representa a instituição para a família. Desse modo, é necessário o aconselhamento e orientação do profissional no processo de suporte social. Além disso, o treinamento em habilidades de suporte social para a parentalidade é uma ferramenta de trabalho para o profissional que contribui no endereçamento das necessidades de suporte social para a parentalidade. É necessário suporte emocional ao profissional que promova a sua regulação emocional. Ademais, para o suporte instrumental possibilitar o fluxo de rede efetivo no território e o encaminhamento responsável da família, também contribui para a qualidade do suporte oferecido e convergência com necessidades que foquem o bem-estar da criança na família. Observou-se também a necessidade de monitoramento social, através de supervisão direta e sistemática, sobre o trabalho de suporte social desenvolvido pelo profissional com funções de suporte.

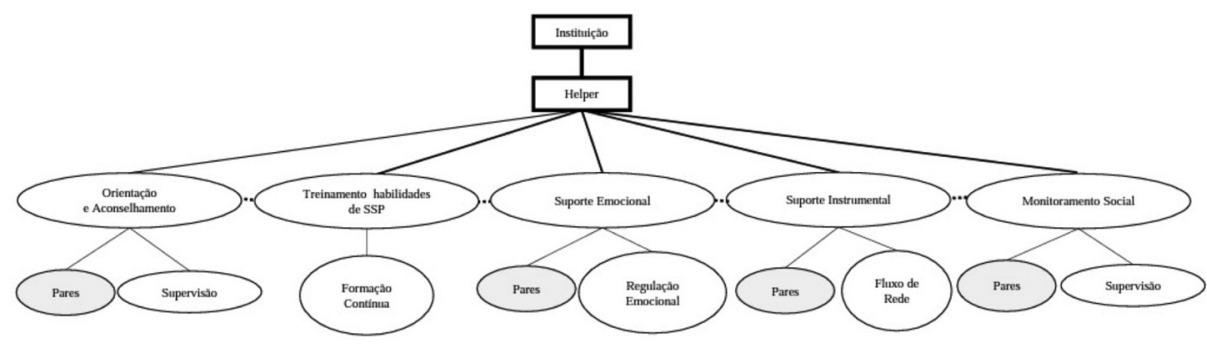

Figura 2. Modelo de suporte social parental.

\section{Discussão dos resultados}

A necessidade identificada de formação de profissionais para o exercício do suporte social parental pode dever-se a diversas razões. Uma primeira razão são 
as diferenças de estatuto socioeconómico entre profissional de suporte e família, as quais parece constituírem valores dissonantes na relação entre o profissional dador e o recetor, seja esse último figura parental ou a criança. Por exemplo, relatos de práticas parentais negligentes ou abusivas foram relacionados, por alguns profissionais, com baixo estatuto socioeconómico familiar e com contexto de violência urbana das famílias em situação de pobreza. Uma segunda razão que indica a necessidade de formação dos profissionais para o suporte social parental são as perceções dos profissionais sobre orientações e aconselhamento ofertados à família. Estudos indicam que inseguranças relacionadas com o exercício parental e divergências sobre as atitudes da criança na escola e no lar não estão relacionadas com a fratria familiar (Nielsen-Berg et al., 2011; Novaes \& Gaspar, 2015; Thompson \& Goodvin, 2015), como citado por alguns profissionais da creche nesta investigação, mas sim a condições de stresse tóxico e fatores de risco no exercício parental. Percebe-se que o suporte social parental formal, quando não há formação específica do profissional para tal, é associado por este a valores e crenças religiosas, constituindo crenças parentais e cognições parentais do indivíduo. Uma terceira razão é a disponibilidade do profissional. Alguns profissionais apresentam maior disponibilidade na relação com a figura parental e essa postura parece estar relacionada com a institucionalização das necessidades de suporte social parental identificadas, ou seja, a identificação das necessidades de suporte social e endereçamento, por meio de intervenção ou encaminhamento, para contenção do stresse vivenciado pela família. A não institucionalização das necessidades de suporte familiar identificadas pelos profissionais converge as ações de suporte para o âmbito informal, o que pode agravar a situação de risco da família e, especialmente, da criança.

Sobre a legislação de suporte à parentalidade no Brasil, neste estudo observa-se o suporte oferecido por leis que contribuem para o exercício parental e manutenção no mercado de trabalho. Uma decisão liminar orienta para a diminuição do horário de trabalho de figura parental de criança com TEA no serviço público (Decisão Liminar Tribunal de Justiça Federal e dos Territórios, n. 20140020331773MSG art. 21, III, da Portaria No 199/2014, Tribunal de Justiça do Distrito Federal e dos Territórios, 2015). Essa observação de necessidade de suporte para famílias com crianças com transtornos de desenvolvimento já foi realizada anteriormente num estudo de Lima et al. (2014), de forma que tal legislação específica relacionada com flexibilidade de horário no trabalho e exercício da parentalidade converge para discussão sobre legislação de suporte à parentalidade no Brasil.

O modelo apresentado na Figura 2 e o endereçamento de treinamento de habilidades parentais para famílias em condições de adversidade, por meio da Figura 1, pretendem colaborar no processo de suporte social ao profissional. Destaca-se também que, no âmbito de implicações para a prática, a lógica do território e 
articulação eficaz do fluxo de rede são ferramentas da intervenção psicossocial em saúde mental que podem contribuir para o estabelecimento e sustentabilidade da relação formal de suporte social entre instituição, criança e figura parental.

Este estudo apresenta limitações tanto devido a sua natureza qualitativa e número de participantes, quanto ao acesso por conveniência às instituições. Desse modo, futuros estudos podem integrar uma abordagem qualitativa e quantitativa no âmbito da implementação de intervenções no contexto brasileiro. Outra possibilidade é o desenvolvimento de estudos quantitativos para mensuração de necessidades de suporte ao profissional na atuação de suporte social à família.

As autoras buscaram identificar possibilidades para inserção de treinamento de habilidades parentais positivas em contexto brasileiro como ferramenta de prevenção em saúde para famílias, em condições de adversidade, em equipamentos utilizados com frequência diária ou semanal. Estudos futuros devem observar alterações na perceção do suporte oferecido e recebido, tanto na perspetiva dos profissionais como dos recetores, antes a após a introdução de treinamento de habilidades parentais positivas em equipamentos de suporte social à família no Brasil. Sendo assim, observa-se que a implementação de intervenções sistemáticas e baseadas em evidência para o desenvolvimento de uma atitude parental positiva podem contribuir para o estabelecimento de uma relação formal de suporte social parental, vinculação da família com a instituição e posterior manutenção do suporte social oferecido em condições de stresse tóxico ou pobreza.

\section{REFERÊNCIAS}

Abreu-Lima, I., Alarcão, M., Almeida, A., Brandão, T., Cruz, O., Gaspar, M., \& Santos, M. (2010). Avaliação de intervenções de educação parental: Relatório 2007-2010. Consultado em http:// www.cnpcjr.pt/preview_documentos.asp?r=3493\&m=PDF

Araújo, A., Amaro, E., Casella, E. B., Cunha, F., Costa, J. C., Oliveira, J. B., ... Schawrtzman, S. (2011). Children's learning: An approach of neuroscience, economy, and cognitive psychology. Rio de Janeiro, Brazil: Brazilian Academy of Science, Science and Technology for National Development, Strategic Studies.

American Psychiatric Association (2013). Diagnostic and statistical manual of mental disorders ( $5^{\text {th }}$ ed.). Washington, DC: American Psychiatric Association. doi: 10.1176/appi. books.9780890425596

Axberg, U., \& Broberg, A. G. (2012). Evaluation of “The Incredible Years” in Sweden: The transferability of an American parent-training program to Sweden. Scandinavian Journal of Psychology, 53(3), 224-232. doi: 10.1111/j.1467-9450.2012.00955.x

Borges, C. C., Magalhães, A. S., \& Féres-Carneiro, T. (2015). Liberdade e desejo de constituir família: Percepções de jovens adultos. Arquivos Brasileiros de Psicologia, 66(3), 89-103. 
Bornstein (2012). Cultural approaches to parenting. Parenting: Science and Practice, 12(2-3), 212-221. doi: 10.1080/15295192.2012.683359

Brasil (2009). Critérios para um atendimento em creches que respeite os direitos fundamentais das crianças. Ministério da Educação. Secretaria de Educação Básica. Brasília: Ministério da Educação. Consultado em http://agendaprimeirainfancia.org.br/arquivos/direitosfundamentais.pdf

Brasil (2013). Linha de cuidado para a atenção às pessoas com transtornos do espectro do autismo e suas famílias na rede de atenção psicossocial do SUS. Ministério da Saúde. Secretaria de Atenção à Saúde. Departamento de Ações Programáticas Estratégicas: Brasília, Ministério da Saúde. Consultado em http://www.otics.org/estacoes-de-observacao/saude-mental/acervo/arquivos/linha-de-cuidado-para-a-atencao-as-pessoas-com-transtornos-do-espectro-do-autismo-e-suas-familias/view

Braum, V., \& Clarke, V. (2006). Using thematic analysis in psychology. Qualitative Research in Psychology, 3(2), 77-101. doi: 10.1191/1478088706qp063oa

Center on the Developing Child at Harvard University (2010). The foundations of life long health. Boston, Massachussets. Consultado em http://46y5eh11fhgw3ve3ytpwxt9r.wpengine.netdna-cdn.com/wp-content/uploads/2010/05/Foundations-of-Lifelong-Health.pdf

Child on Europe Report (2011). The impact of the economic crisis on children: Lessons from the past experiences and future policies. The proceedings of the Child on Europe Seminar on the impact of the crisis on children. (Report 2011). Secretary Child on Europe. Firenze, Itália: Italian Childhood and Adolescence Documentation and Analysis Centre, Istituto degli Innocenti.

Child on Europe Report (2013). Public policies supporting positive parenthood: New policies perspectives. The proceedings of the Child on Europe Seminar on positive parenthood. (Report 2013). Secretary Child on Europe. Firenze, Itália: Italian Childhood and Adolescence Documentation and Analysis Centre, Istituto degli Innocenti. Consultado em http://www.childoneurope.org/ issues/publications/COEseries8-Positiveparenthood.pdf

Colin, D. R., Pereira, J. M., \& Gonelli, V. M. (2013). Construction trajectory of integrated management of unified social assistance system, unique cadastre, and Bolsa Família program for the consolidation of Brazilian model of social protection. In T. Campello \& M. C. Neri (Org.), Bolsa Família program: A decade of inclusion (pp. 47-64). Brasília: Institute of Economic and Applied Research, Ipea.

Cruz, O. (2005). Parentalidade. Coimbra: Quarteto.

Cunha, F., \& Heckman, J. J. (2007). The technology of skill formation. American Economic Review, 97(2), 31-37. doi:10.1257/aer.97.2.31

Decisão Liminar Tribunal de Justiça Federal e dos Territórios (2015). n. 20140020331773MSG art. 21, III, da Portaria No 199/2014, Tribunal de Justiça do Distrito Federal e dos Territórios. Consultado em http://www.tjdft.jus.br/institucional/imprensa/noticias/2015/junho/mae-de-portador-de-deficiencia-tem-direito-a-jornada-reduzida-sem-compensacao

Doyle, O., Harmon, C. P., Heckman, J. J., \& Tremblay, R. E. (2009). Investing in early human development: Timing and economic efficiency. Economics and Human Biology, 7, 1-6. doi: 10.1016/j. ehb.2009.01.002

Harkness, S., \& Super, C. M. (2002). Culture and parenting. In M. H. Bornstein (Ed.), Handbook of parenting: Biology and ecology of parenting (Vol. 2, pp. 253-280). Mahwah: Lawerence Erlbaum Associates.

Hutchinson, A. J., Johnson, L. H., \& Breckon, J. D. (2010). Using QRS-NVivo to facilitate the development of a grounded theory project: An account of worked example. International Journal of Social Research Methodology, 13(4), 283-302. doi: 10.1080/13645570902996301 
Lima, R. C., Couto, M. C., Delgado, P. G., \& Oliveira, B. D. (2014). Indicadores sobre o cuidado a crianças e adolescentes com autismo na rede de CAPSi da região metropolitana do Rio de Janeiro. Physis, 24(3), 715-739. doi: 10.1590/S0103-73312014000300004

McGilloway, S., Mhaille, G. N., Bywater, T., Furlong, M., Leckey, Y., Kelly, P., ... Donelly, M. (2012). A parenting intervention for childhood behavioral problems: A randomized controlled trial in disadvantaged community-based settings. Journal of Consulting and Clinical Psychology, 80(1), 116-127. doi: 10.1037/a0026304

Miller, W., \& Crabtree, B. F. (2004). Depth interviewing. In S. N. Hesse-Biber \& P. Levy (Org.), Approaches to qualitative research: A reader on theory and practice (pp. 185-202). New York: Oxford University Press.

Morawska, A., Sanders, M., Goadby, E., Headley, C., Hodge, L., McAuliffe, C., ... Anderson, E. (2011). Is the Triple P-positive parenting program acceptable to parents from culturally diverse backgrounds? Journal of Child and Family Studies, 20(5), 614-622. doi: 10.1007/s10826-010-9436-x

Nielsen-Berg, T. S., Solhein, E., Belsky, J., \& Wichstrom, L. (2011). Preschoolers' psychosocial problems: In the eyes of the beholder? Adding teacher characteristics as determinants of discrepant parent-teacher reports. Child Psychiatry Human Developmet, 43(3), 393-413. doi: 10.1007/ s10578-011-0271-0

Novaes, M. B. (2015). Educação parental e prevenção intersetorial em saúde pública: A promoção da parentalidade positiva no Brasil e em Portugal. (Tese de doutorado não publicada). Universidade de Coimbra, Coimbra.

Novaes, M. B., \& Gaspar, M. F. (2015, September). Social support and parenting in Brazil: Representative perspectives of the protective welfare system to childhood and family. Poster communication presented at the $17^{\text {th }}$ European Conference on Developmental Psychology, Braga, Portugal.

Pêcheux, M. (1983). O discurso: Estrutura ou acontecimento. Campinas: Ponte Editores.

Raikes, H. A., \& Thompson, R. A. (2005). Efficacy and social support as predictors of parenting stress among families in poverty. Infant Mental Health Journal, 26(3), 177-190. doi: 10.1002/imhj.20044

Ramos, D., Seidl-de-Moura, M. L., \& Pessoa, L. F. (2013). Achievement goals of youngsters in Rio de Janeiro in different contexts. Paidéia, 23(56), 321-328. doi: 10.1590/1982-43272356201306

Rank, M. (2004). The blending of qualitative and quantitative methods in understanding childbearing among welfare. In S. N. Hesse-Biber \& P. Levy (Org.), Approaches to qualitative research: A reader on theory and practice (pp. 81-96). New York: Oxford University Press.

Respler-Herman, M., Mowder, B. A., Yasik, A. E., \& Shamah, R. (2012) Parenting beliefs, parental stress, and social support relationships. Journal of Child and Family Studies, 21(2), 190-198. doi: 10.1007/s10826-011-9462-3

Thompson, R. A. (1995). Preventing child maltreatment through social support: A critical analysis. Thousand Oaks, CA: Sage.

Thompson, R. A. (2011). Attachment relationships in early head start families. Attachment \& Human Development, 13(1), 91-98. doi: 10.1080/14616734.2010.503578

Thompson, R. A. (2015). Social support and child protection: Lessons learned and learning. Child Abuse \& Neglect, 41, 19-29. doi: 10.1016/j.chiabu.2014.06.011

Thompson, R. A., \& Goodvin, R. (2015). Social support and developmental psychopatology. In D. Ciccheti (Org.), Developmental Psychopathology (3 ${ }^{\text {rd }}$ ed.). New York: Wiley. doi: 10.1002/9780470939406.ch1

Zimmerman, M., \& Sprague, J. (2004). Overcoming dualisms: A feminist agenda for sociological methodology. In S. N. Hesse-Biber \& P. Levy (Org.), Approaches to qualitative research: A reader on theory and practice (pp. 39-61). New York: Oxford University Press. 